\title{
Activity Estimation Method of Linear Gamma-Ray Source under Shield Based on Single Compton Scattering
}

\author{
Maolin Xiong, Jin Yang, Xian Guan \\ Sichuan Province Key Laboratory of Applied Nuclear Techniques in Geosciences, Chengdu University of Technology, Chengdu, \\ China \\ Email: mlxiong94@163.com
}

How to cite this paper: Xiong, M.L., Yang, J. and Guan, X. (2020) Activity Estimation Method of Linear Gamma-Ray Source under Shield Based on Single Compton Scattering. Open Access Library Journal, 7: e6557. https://doi.org/10.4236/oalib.1106557

Received: June 25, 2020

Accepted: July 19, 2020

Published: July 21, 2020

Copyright $\odot 2020$ by author(s) and Open Access Library Inc.

This work is licensed under the Creative

Commons Attribution International

License (CC BY 4.0).

http://creativecommons.org/licenses/by/4.0/

(c) (i) Open Access

\begin{abstract}
In nuclear emergencies, it is necessary to estimate the activity of radioactive rapidly and efficiently. This paper proposes an activity estimation method of linear gamma-ray source based on the single Compton scattering of gamma-ray. The paper establishes a physics model of radiation detection, and deduces the relation of the full-energy peak count and the single Compton scattering count. Based on the physics model and the relation of the full-energy peak and single Compton scattering count, the activity estimation method of linear gamma-ray source is built, which can be applied to different shield and the radioactivity of the linear gamma-ray source can be estimated rapidly and efficiently.
\end{abstract}

\section{Subject Areas}

Nuclear Physics

\section{Keywords}

Activity Estimation, Compton Scattering, Nuclear Emergency,

Numerical Calculation

\section{Introduction}

Currently, radioactive sources are widely used in various fields, such as medical, industrial, agricultural, health, scientific research (F. Abdollah Mianji, 2007) [1]. There are about 84,000 disused radioactive sources accumulated in China (Sheng Feng, 2018) [2]. These sources have a potential risk of loss, and they need to routine inspection, which will be a risk factor and workload for functional depart- 
ment.

When disposing the disused and lost radioactive sources, we need to make a preliminary estimation of the radioactivity of the source, firstly. But it is a difficult work, when the source is under shield which thickness usually is unknown. Ajith Gunatilaka et al. (2010) [3] utilized a low-cost Geiger-Muller counter to estimate the parameters of radioactive sources (their radioactivity and location), the experimental results show that the approach performs well when no more than two sources are present. Budhaditya Deb et al. (2010) [4] derive Maximum Likelihood based source estimation algorithms for a system of Non-Directional (CsI based scintillation detectors) and Directional (CZT based Compton detectors) radiation detectors, results are presented which illustrate the behavior and performance of the approach in a single source. D. Ridikas et al. (2005) [5] proposed a non-destructive method of characterization of radioactive waste containers using spectroscopy and Monte Carlo techniques, and quantified the limits and uncertainties of the proposed method for the activity estimates in the case of ${ }^{137}$ Cs as a tracer element. S. N. P'ya et al. (2015) [6] used a xenon gamma spectrometer to determine the radioactivity of radioactive waste in containers, the result shows that the XGS has good capabilities for nuclide identification and their activity estimation. Yann Sepulcre et al. (2013) [7] presented a method based on sparse representation of a sampled spectrometric signal to estimate the activity of an unknown radioactive source, results on simulations and real data both emphasize the efficiency of the method. Morelande et al. (2008) [8] analyzed the estimation problem for multiple radioactive sources using maximum likelihood estimation and Bayesian estimation, the results show that the Bayesian approach is capable of excellent performance with reasonable computational expense. Jifu Zhao et al. (2018) [9] used the kernel density estimation (KDE) and grid search with maximum likelihood estimation (MLE) to estimate the source intensity, and simulation study and experiment are conducted to compare the effectiveness of the proposed methods.

Activity estimation method of linear gamma-ray source is proposed in this paper, which estimates the activity of the source and the thickness of the shield by deducing the relation of the full-energy peak count and the single Compton scattering count, and it can be applied to estimate the activity of linear gamma-ray source under different structures and material shield, efficiently and reliably.

\section{The Activity Estimation Method}

In general, to be sure radiation safety, the linear gamma-ray source is under shield, and the common structure of shield is box, cylinder and sphere. In nuclear emergencies, the thickness of the shield and the activity and the length of the linear gamma-ray source are all unknown, so the gamma-ray spectrometry is the most effective means to quickly estimate the thickness of the shield and activity of gamma-ray. The physics model of the activity estimation method is established, as shown in Figure 1. 


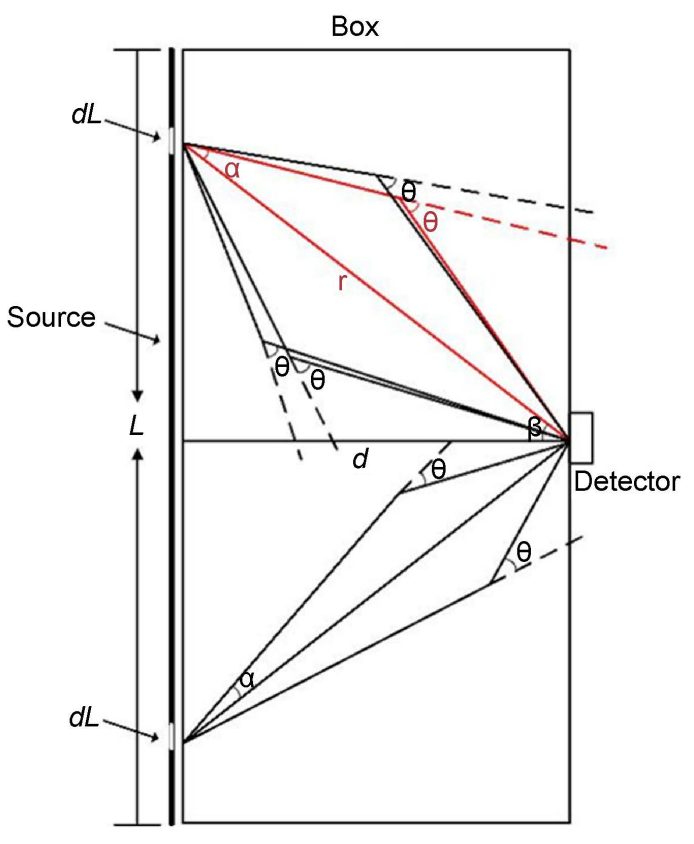

(a)

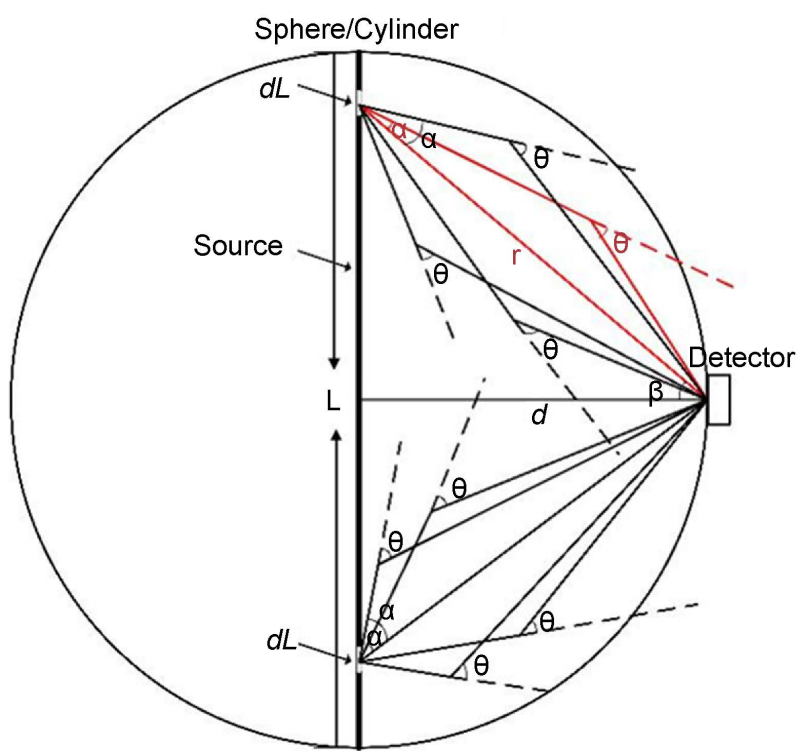

(b)

Figure 1. The physics model of radioactivity estimation method.

As Figure 1, the shield of part (a) is a box, the shield of part (b) is a cylinder or sphere. The gamma-ray detectors and linear gamma-ray source are closed to the surface of the shield. The center of the linear gamma-ray source and the detector are on the horizontal line, and the detector is perpendicular to the linear source. Due to the thickness of the shield and the radioactivity and the length of the linear gamma-ray source are all unknown, the length of the linear gamma-ray source is assumed that same to height or diameter of the shield.

If the linear gamma-ray source is not under shield, the intensity $\phi_{0}$ of gamma-ray into detector is given as follows, 


$$
\phi_{0}=\int_{L} \frac{\Omega}{4 \cdot \pi \cdot L} \cdot A \cdot \eta \cdot \lambda \cdot \mathrm{d} L
$$

where, $\Omega$ is the solid angle of the detector to the $\mathrm{d} L, A$ is the radioactivity of the source, $\eta$ is the emission ratio of the gamma-ray, $\lambda$ is linear density of the source, $L$ is the length of the source.

As shown in Figure 1, if the linear gamma-ray source is under the shield. When the gamma-ray goes through the shield, the gamma-ray will be attenuated according to the Beer-Lambert's law (Elmahroug et al. 2013) [10]. The characteristic gamma-ray flux $\phi$ into detector is given as follows,

$$
\phi=2 \cdot \int_{\beta} \frac{\phi_{0}}{L} \cdot \mathrm{e}^{-\mu_{1} \cdot \frac{d}{\cos \beta}} \cdot d \cdot \sec ^{2} \beta \cdot \mathrm{d} \beta
$$

where, $\mu_{1}$ is the linear attenuation coefficient of the shielding material, $d$ is the thickness of the shield, $\beta$ is the angle of entering detector.

Similarly, if the gamma-ray source is under the shield, when the gamma-ray goes through the shield and the Compton Scatter occurs, as shown in Figure 1. The gamma-ray flux $\phi_{s}$ into the detector, which cause by the single scattering ray with a certain scattering angle, is given as follows,

$$
\begin{aligned}
& \phi_{s}=\frac{1}{\pi} \cdot \phi \cdot \Sigma(\theta) \cdot \int_{\beta} \iint_{\Omega} \mathrm{e}^{-\mu_{1} \cdot \frac{d \cdot\left(\cos \alpha-\frac{\sin \alpha}{\tan \theta}\right)}{\cos \beta}} \cdot \mathrm{e}^{-\mu_{2} \cdot \frac{d \cdot \sin \alpha}{\cos \beta \cdot \sin \theta}} \cdot d \cdot \sec ^{2} \beta \cdot \sin \alpha \cdot \mathrm{d} \alpha \cdot \mathrm{d} \varphi \cdot \mathrm{d} \beta \\
& \phi_{s}=2 \cdot \phi \cdot \Sigma(\theta) \cdot \int_{\beta} \int_{0}^{\theta} \mathrm{e}^{-\mu_{1} \cdot \frac{d \cdot\left(\cos \alpha-\frac{\sin \alpha}{\tan \theta}\right)}{\cos \beta}} \cdot \mathrm{e}^{-\mu_{2} \cdot \frac{d \cdot \sin \alpha}{\cos \beta \cdot \sin \theta}} \cdot d \cdot \sec ^{2} \beta \cdot \sin ^{\alpha} \cdot \mathrm{d} \alpha \cdot \mathrm{d} \beta
\end{aligned}
$$

where, $\Sigma(\theta)$ is the probability that gamma-ray is scattered in shield material through unit length with the $\theta$ scattering angle, $\mu_{2}$ is the linear attenuation coefficient of the shielding material when the gamma-ray is scattered with the $\theta$ scattering angle, $\alpha$ is the angle of incidence.

The full-energy peak count of characteristic gamma-ray in the spectrum can be represented as follows,

$$
I=2 \cdot \int_{\beta} \frac{\phi_{0}}{L} \cdot \mathrm{e}^{-\mu_{1} \cdot \frac{d}{\cos \beta}} \cdot d \cdot \sec ^{2} \beta \cdot \mathrm{d} \beta \cdot \varepsilon_{1} \cdot t
$$

where, $I$ is the full-energy peak net count, $\varepsilon_{1}$ is the intrinsic full-energy peak detection efficiency of the detector, $t$ is the measurement time.

Similarly, the count of single scattering ray with a scattering angle $\theta$ in the spectrum can be represented as follows,

$$
I_{\mathrm{s}}=2 \cdot \phi \cdot \Sigma(\theta) \cdot \int_{\beta} \int_{0}^{\theta} \mathrm{e}^{-\mu_{1} \cdot \frac{d \cdot\left(\cos \alpha-\frac{\sin \alpha}{\tan \theta}\right)}{\cos \beta}} \cdot \mathrm{e}^{-\mu_{2} \cdot \frac{d \cdot \sin \alpha}{\cos \beta \cdot \sin \theta}} \cdot d \cdot \sec ^{2} \beta \cdot \sin ^{\alpha} \cdot \mathrm{d} \alpha \cdot \mathrm{d} \beta \cdot \varepsilon_{2} \cdot t
$$

where, $I_{\mathrm{s}}$ is the net scattered count, $\varepsilon_{2}$ is the intrinsic detection efficiency of scattered ray, $t$ is the measurement time.

When the gamma spectrum is recorded by the detector, the thickness of the 
shield can be represented as follows,

$$
\frac{I}{I_{\mathrm{s}}}=\frac{\int_{\beta} \frac{1}{L} \cdot \mathrm{e}^{-\mu_{1} \cdot \frac{d}{\cos \beta}} \cdot d \cdot \sec ^{2} \beta \cdot \mathrm{d} \beta \cdot \varepsilon_{1}}{\Sigma(\theta) \cdot \int_{\beta} \int_{0}^{\theta} \mathrm{e}^{-\mu_{1} \cdot \frac{d \cdot\left(\cos \alpha-\frac{\sin \alpha}{\tan \theta}\right)}{\cos \beta}} \cdot \mathrm{e}^{-\mu_{2} \cdot \frac{d \cdot \sin \alpha}{\cos \beta \cdot \sin \theta}} \cdot d \cdot \sec ^{2} \beta \cdot \sin ^{\alpha} \cdot \mathrm{d} \alpha \cdot \mathrm{d} \beta \cdot \varepsilon_{2}}
$$

As shown in Figure 1, when the gamma-ray goes through the shield, the ray interacts with shielding material and photoelectric effect, pair effect or Compton scattering occur randomly. The count which is recorded by detector depends on the thickness of the shield, the radioactivity of the source and the detection efficiency of the detector. Therefore, the equation for the thickness of the shield and counts is established, as shown in Equation (6). When the thickness of the shield is known, the radioactivity of the source can be calculated quickly by Equations (1) and (4).

Based on the model assumptions and associated equations, a radioactivity estimation method can be developed as follows:

(1) Use the WinXCOM code (Gerward et al., 2004) [11] to calculate $u_{1}, u_{2}$, respectively,

(2) Determine the scattering angle $\theta$,

(3) Calculate $\Sigma(\theta)$ as below,

$$
\Sigma(\theta)=\frac{\mathrm{d} \sigma}{\mathrm{d} \Omega} \cdot n \cdot Z
$$

where, $\frac{\mathrm{d} \sigma}{\mathrm{d} \Omega}$ is the differential cross-section, $n$ is the nucleon density, $Z$ is the atomic number of shielding material,

(4) Use the Monte Carlo code MCNP5 to calculate $\varepsilon_{1}$ and $\varepsilon_{2}$, respectively,

(5) Calculate the thickness $d$ of the shield by Equation (6),

(6) Calculate the radioactivity $A$ of the source by Equations (4) and (1).

\section{Conclusion}

In this article, we propose a radioactivity estimation method of the disused and lost linear radioactive source, this method congregates the advantages of the numerical calculation and Monte Carlo simulation. The numerical calculation shows that the method can be used in nuclear emergencies, and help us reduce the risk factor and workload in disposing the disused and lost radioactive source. However, it still needs further discussion for practical applications due to the influence of detector type, the type of radioactive source, etc.

\section{Acknowledgements}

I would like to give my sincere gratitude to my supervisor Mr. Zhang, who gave me great help during the whole process of my thesis writing. Thanks to his patience, kindness, encouragement and good advice, I can successfully finish my paper. 


\section{Conflicts of Interest}

The authors declare no conflicts of interest regarding the publication of this paper.

\section{References}

[1] Mianji, F.A. (2007) Analyzing an Accident Related to Orphan Sources Including Dose Assessment. Journal of Radiation Protection Dosimetry, 123, 394-397. https://doi.org/10.1093/rpd/ncl147

[2] Feng, S. (2017) Disposal Status of Disused Radioactive Sources in France. Journal of Environment \& Development, 9, 194-195.

[3] Gunatilaka, A., Ristic, B. and Morelande, M. (2010) Experimental Verification of Algorithms for Detection and Estimation of Radioactive Sources. 13th Conference on Information Fusion, Edinburgh, 26-29 July 2010, 1-8. https://doi.org/10.1109/ICIF.2010.5711880

[4] Budhaditya, D., Ross, J.A.F., Ivan, A. and Hartman, M.J. (2010) Radioactive Source Estimation using a System of Directional and Non-Directional Detectors. IEEE Transactions on Nuclear Science, 58, 3281-3290. https://doi.org/10.1109/TNS.2011.2165558

[5] Ridikas, D., et al. (2005) Non-Destructive Method of Characterization of Radioactive Waste Containers Using Gamma Spectrometry and Monte Carlo Techniques. Journal of Radiation Protection Dosimetry, 115, 113-116. https://doi.org/10.1093/rpd/nci253

[6] P'ya, S.N., et al. (2015) Determination of Radioactive Waste Activity in Containers Using a Xenon Gamma Spectrometry. Bulletin of the Lebedev Physics Institute, 42, 249-253. https://doi.org/10.3103/S1068335615080072

[7] Sepulcre, Y., et al. (2013) Sparse Regression Algorithm for Activity Estimation in $\gamma$ Spectrometry. IEEE Transactions on Signal Processing, 61, 4347-4359.

https://doi.org/10.1109/TSP.2013.2264811

[8] Morelande, M., Ristic, B. and Gunatilaka, A. (2007) Detection and Parameter Estimation of Multiple Radioactive Sources. Proceedings of the 10 th International Conference on Information Fusion, Quebec, 9-12 July 2007, 1-7. https://doi.org/10.1109/ICIF.2007.4408094

[9] Zhao, J.F. and Sullivan, C.J. (2018) Detection and Parameter Estimation of Radioactive Sources with Mobile Sensor Networks. Journal of Radiation Physics and Chemistry, 155, 265-270. https://doi.org/10.1016/j.radphyschem.2018.06.018

[10] Elmahroug, Y., et al. (2013) Calculation of Gamma and Neutron Shielding Parameters for Some Materials. J. International Journal of Physics and Research, 3, 33-40.

[11] Gerward, L., Guilbert, N., et al. (2004) WinXcom: A Program for Calculating X-Ray Attenuation Coefficients. Radiation Physics and Chemistry, 71, 653-654. https://doi.org/10.1016/j.radphyschem.2004.04.040 